\title{
What Parameters Correlate with the Sense of Knowledge During the Early Phase of a Bird Flu Outbreak in Israel?
}

\author{
Galit Shohat ${ }^{1}$, Rami Peltz ${ }^{1}$ and Yaron Bar-Dayan ${ }^{*}, 1,2$ \\ ${ }^{I}$ IDF Home Front Command, Israel \\ ${ }^{2}$ Faculty of Health Sciences, Ben Gurion University, Beer-Sheva, Israel
}

\begin{abstract}
Introduction: On March 2006, 298,000 cases of birds infected with bird flu were destroyed in nine rural settlements in Israel, out of a total of 1.2 million birds that were destroyed within these settlements and in a radius of $3 \mathrm{~km}$. The population was instructed to take precautionary measures to prevent a disease outbreak. The sense of knowledge of the population concerning the disease correlated with compliance with authorities' instructions.

Objectives: To analyze the relationships between the sources of information, and public emotions and interest in avian influenza with the sense of knowledge concerning the disease in the affected area and the nationwide population, during the first phase of a bird flu outbreak in Israel.

Methods: We conducted a telephone survey among two randomly selected, representative samples of adults, during the first phase of a bird flu outbreak. One sample involved 500 adult residents of the nationwide area; and the other sample involved 103 adult residents of the affected area. We measured the use of mass media, emotions, interest, and sense of knowledge of the population concerning avian influenza. We analyzed the relationships between the sources of information and public emotions with the sense of knowledge using chi-square and t-tests. A P value of $<0.05$ was considered to be statistically significant.

Results: TV viewers in the affected area had a significantly lower sense of knowledge concerning avian influenza $(\mathrm{P}<0.05)$. Internet users in the nationwide population had a significantly higher sense of knowledge $(\mathrm{P}<0.05)$. People in the affected area with higher levels of stress had a significantly lower sense of knowledge $(\mathrm{P}<0.05)$. People in the nationwide population with a higher level of interest had a significantly higher sense of knowledge $(\mathrm{P}<0.05)$.

Conclusion: The use of the internet as a source of information, combined with a high level of interest and low levels of stress correlated with a high sense of knowledge concerning avian influenza during the early phase of an avian influenza outbreak in Israel. Authorities should consider these findings when planning the strategy of mass media use, in order to increase the public's sense of knowledge concerning the disease and to enhance control of the disease outbreak by improving the compliance of the population with the authorities' instructions.
\end{abstract}

\section{INTRODUCTION}

It is widely anticipated that a global H5N1 avian influenza pandemic will occur in the near future. Concerns regarding the disease were raised in recent years following reports that this new, highly pathogenic strain of avian influenza, was spreading across Asia, infecting both poultry and people [1]. Infectious diseases experts are concerned that a mutation of the virus could result in higher infectivity and pathogenicity via human to human transmission and estimated that it may cause as many as 7.4 million deaths [2]. Of the 317 people known to be infected by the end of June 2007, 191 had lost their lives. There may have been others who were never found, diagnosed, reported, or had subclinical disease [3].

Effective public health action depends on the public's psychological responses and the prevailing perceptions and beliefs of the community [4]. It is very likely, that even at

*Address correspondence to this author at the Department of Disaster and Emergency Medicine, The Faculty of Health Sciences, Ben Gurion University, Beer-Sheva, Israel; 16 Dolev St. Neve Savion, Or-Yehuda, Israel; Tel: 00972578186215; E-mail: bardayan@netvision.net.il the onset of a human avian influenza epidemic, widespread distress, panic and avoidance behaviors would occur in the affected communities [5]. Unconfirmed beliefs and misconceptions about newly emerging infectious disease are inevitable [6]. Raising population anxiety levels through warnings about a disease, produces only transient, inconsistent and therefore, often ineffective results [7].

Media coverage of infectious disease can be a doubleedged sword [8]. On the positive side, news media inform people about how a disease is spread and what precautions to take. On the other hand, because of national and international news coverage of an outbreak, the public might become unduly alarmed. Those who are distant from the site of the outbreak can become concerned and start taking precautions as if they were in the affected area. Informing people who are far from the immediate area of concern might also have a positive affect in that they can begin to take timely precautions should the disease spread [8]. In Singapore [9] despite more than two months of intensive SARS public education, deficiencies in knowledge and behavior persisted. SARS public education through the mass media was important (forms of mass media were the most important sources of 
SARS information) but it remained inadequate. In this case, television, newspaper, and radio formed the top three sources of information on SARS. Aa survey in Hong Kong, revealed substantial misinformation and false beliefs despite heavy and constant mass media and public service announcements [4].

During March 2006, a total of 1.2 million birds were destroyed in nine rural settlements in Israel, where infected birds were found, and in a radius of $3 \mathrm{~km}$. Of 1.2 million birds that were destroyed, 298,000 tested positive for bird flu. No human case of avian influenza was found in this outbreak. The public was instructed by the authorities to take defensive measures to prevent infection [10]. Their sense of knowledge concerning avian influenza correlated with the population's compliance to authorities' instructions [11].

Little is known about the relationships between the use of different forms of mass media and public emotions and the sense of knowledge of the population about avian influenza during the early phase of a bird flu outbreak.

The objective of the paper was to analyze the relationships between the source of information and public emotions and the sense of knowledge of the population concerning avian influenza in the affected area and in the nationwide population during the first phase of a bird flu outbreak in Israel.

\section{METHODS}

\section{Study Population and Sampling}

The data reported in this article are derived from a survey conducted in March 2006, during the early period of an avian influenza outbreak in Israel.

The survey was conducted by telephone with randomly selected, representative samples of adults. Coverage of national telephone ownership in Israel is $85.1 \%$. Trained interviewers conducted anonymous telephone interviews using a structured questionnaire; the median duration of the interviews was four minutes. The entire survey was conducted during one evening in March 2006, from 16:00 to 21:00.

The survey sampled two different populations: one sample involved 500 adult residents of the nationwide area; and the other sample involved 103 adult residents of the affected area.

The sampling frame of the nationwide area is $2,396,370$ households (including the affected area, which is $0.07 \%$ from the nationwide population). Because of the negligible size of the affected area in relation to the nationwide sample, it was assumed that the nationwide sample actually resembled the unaffected area of the country. The sampling frame for the affected area was 1,665 households. The affected area frame sampling was clearly and completely defined by including all settlements within a radius of $3 \mathrm{~km}$, based on the definition of the Ministry of Health.

The sampling frame for both samples was determined by "Dvash", which is a computerized application for locating and randomly sampling phone subscribers out of the total telephone ownership. It is in wide use with the research industry.
Adults (persons 18 years of age or older) who were at home when we called were eligible for the study. If two or more adults were at home, we randomly selected one to interview according to a computerized random choice schedule. At the end of the interview period, 13 percent of the telephone numbers were determined to be nonworking, a fax machine, or otherwise ineligible; 34.2 percent were unanswered after several attempts. Verbal informed consent was obtained from the respondents. Seventeen percent of those who answered phones refused to be interviewed. At the end of the interview period, we completed 603 interviews.

The size of the sampling error was $\pm 4.5 \%$ for the nationwide sample and $\pm 9.8 \%$ for the affected area.

\section{Instrument and Key Measures}

A questionnaire was developed by the head of the Population Behavior Research Branch of the Home Front Command that used questionnaires from previous studies conducted by the branch. The questionnaire was developed with the assistance of a group of physicians from the Medical Department of the Home Front Command, experienced in disaster preparedness.

To assess the sources of information, public emotions, interest and sense of knowledge during the early phase of an avian influenza outbreak, we selected and developed questionnaire items based on the population behavior research branch of the home front command and current media reports. A pilot study with 3 questionnaires was conducted to validate the questions and the questionnaire was revised based on the results of the pilot study. Background variables including age, gender, marital status, education, place of birth and religion were measured.

The study was introduced by the following: "Hello, I am $<$ NAME $>$ from the IDF Home Front Command. We are conducting a short survey to assess public opinion regarding the bird flu outbreak. We would like to ask you a few questions. Your reply will be kept discrete and anonymous and will be used for research purposes only. Do you kindly agree to participate in the survey?" No incentive for participation was offered to the respondents.

The extent of use of each type of mass media as the source of information about avian influenza was measured by the question: "To what extent did you use each form of the following mass media as a source of information concerning bird flu: TV, radio, newspaper, internet". The values for this variable ranged from 1 to 5 , with low values indicating lower use and high values indicating higher use.

The level of feelings towards the bird flu outbreak was measured by the question: "To which extent do you have the following feelings as a result of the bird flu outbreak publication: fear, indifference (listlessness, disinterested), stress and hope. Each of these parameters ranged from 1 to 5, with a low value indicating low levels of feeling towards bird flu and a high value indicate high levels of each feeling.

Interest in the disease was measured by the question: "To what extent are you interested in bird flu?". The values for this variable ranged from 1 to 5 , with low values indicating low interest and high values indicating high interest. 
The sense of knowledge was measured by the question: "To what extent do you feel you have enough information about the bird flu?". The values for this variable ranged from 1 to 5 , with low values indicating low sense of knowledge and high values indicating high sense of knowledge.

For the purpose of analysis, we dichotomized the responses to all questions into two groups: those who answered 4-5 were considered "high" and those who answered 1-3 were considered "low".

\section{Statistical Analysis}

We analyzed the relationship between the source of information, public emotions, and interest of the population in avian influenza with the sense of knowledge of the population concerning the disease. We report the results of univariate analyses (means and percentages) and bivariate analyses (t-tests, and chi-square tests). We used SPSS for windows (version 11.1) and a $P$ value of $<0.05$ was considered statistically significant.

\section{RESULTS}

The demographic characteristics of the two samples are reported in Table 1. There were no significant differences between both samples in gender, age, marital status, or level of education. There were significant differences between the two samples in religion and being born in Israel. The affected area, which is rural only, is populated by a significantly higher percentage of people who were born in Israel and is exclusively populated by a Jewish population compared to the nationwide sample, which is mainly urban.

Participants in the nationwide population who indicated that they received information from the internet had a significantly higher sense of knowledge concerning avian influenza than participants who did not receive information through this source (3.5 compared to $3.2, \mathrm{P}<0.05)$. Participants in the affected area who indicated that they received information from $\mathrm{TV}$ had a significantly lower sense of knowledge concerning avian influenza than participants who

Table 1. Demographic Characteristics of the Two Samples

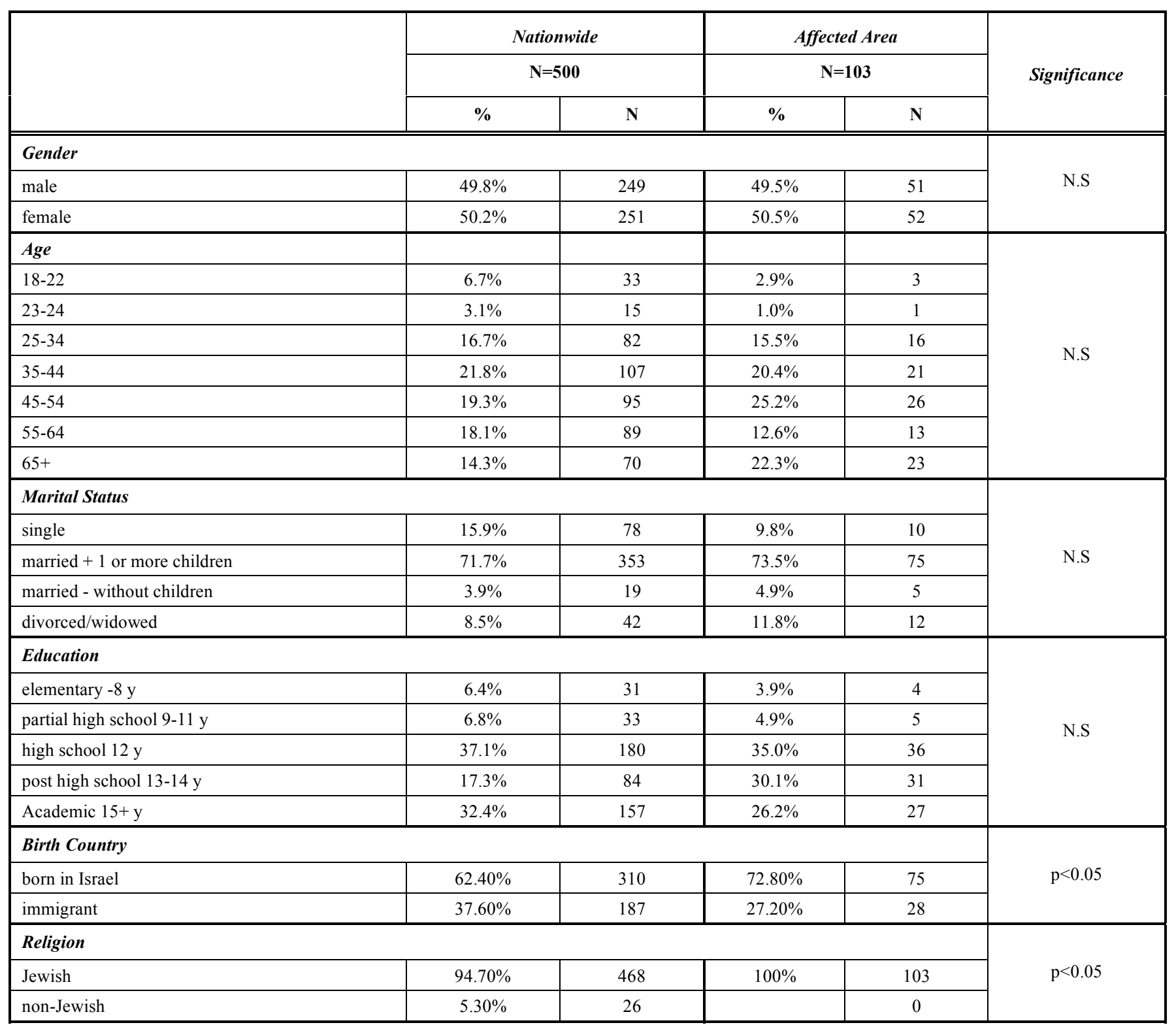


did not receive information through this source (3.3 compared to 3.8, $\mathrm{P}<0.05$ ) (Fig. 1).

People with a high level of interest in avian influenza had a significantly higher sense of knowledge from 3.0 to 3.3 $(\mathrm{P}<0.05)$ in the nationwide population. People with high levels of stress had a significantly lower sense of knowledge from 3.7 to 2.9 in the affected area (Fig. 2).

\section{DISCUSSION}

In this study, we found that the use of internet to collect information, as well as a high level of interest and low levels of stress correlated with a high sense of knowledge concerning avian influenza during the early phase of avian influenza outbreak in Israel.

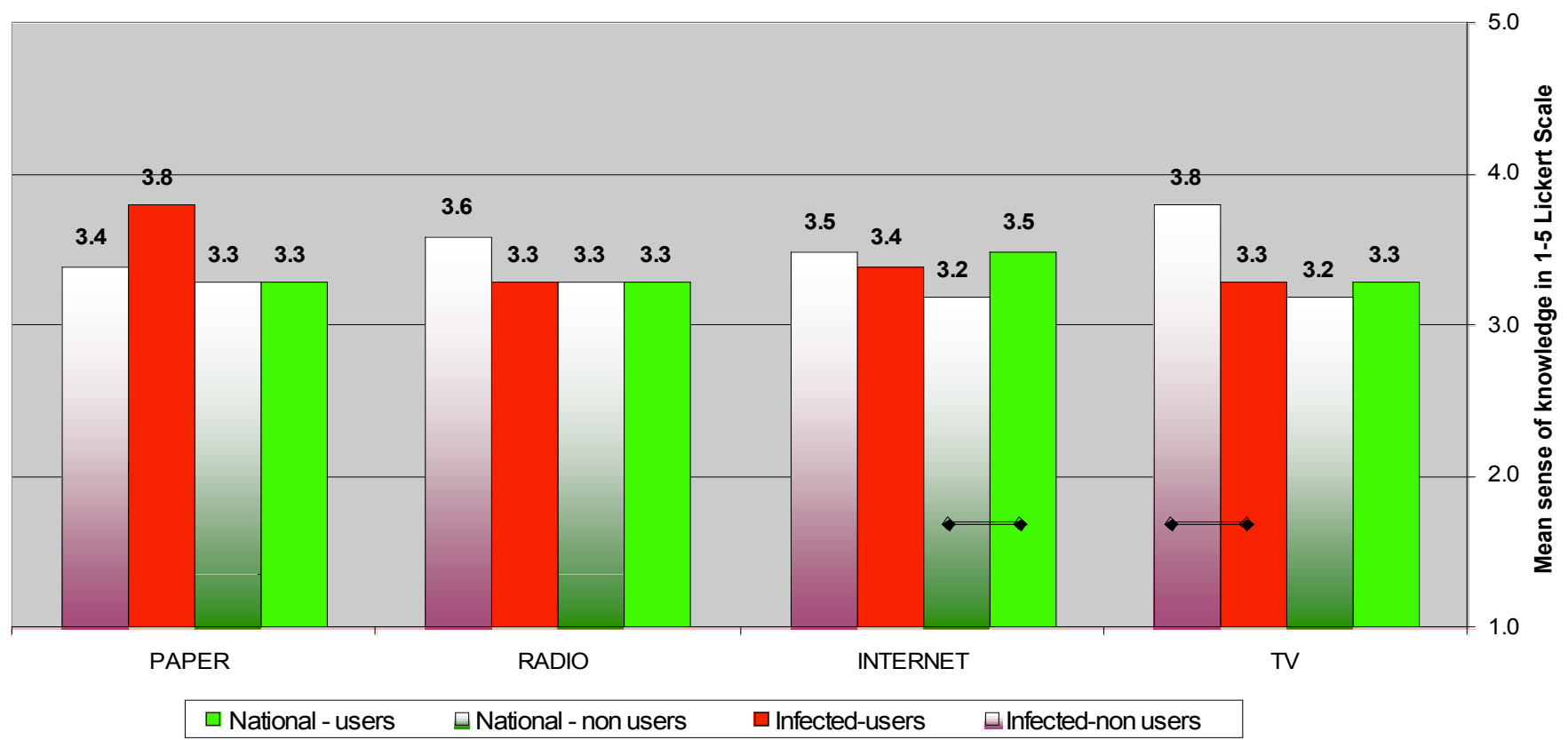

Fig. (1). The effect of different media use on the sense of knowledge concerning bird flu among a representative randomly selected samples of adults in the infected areas and non infected areas in Israel.

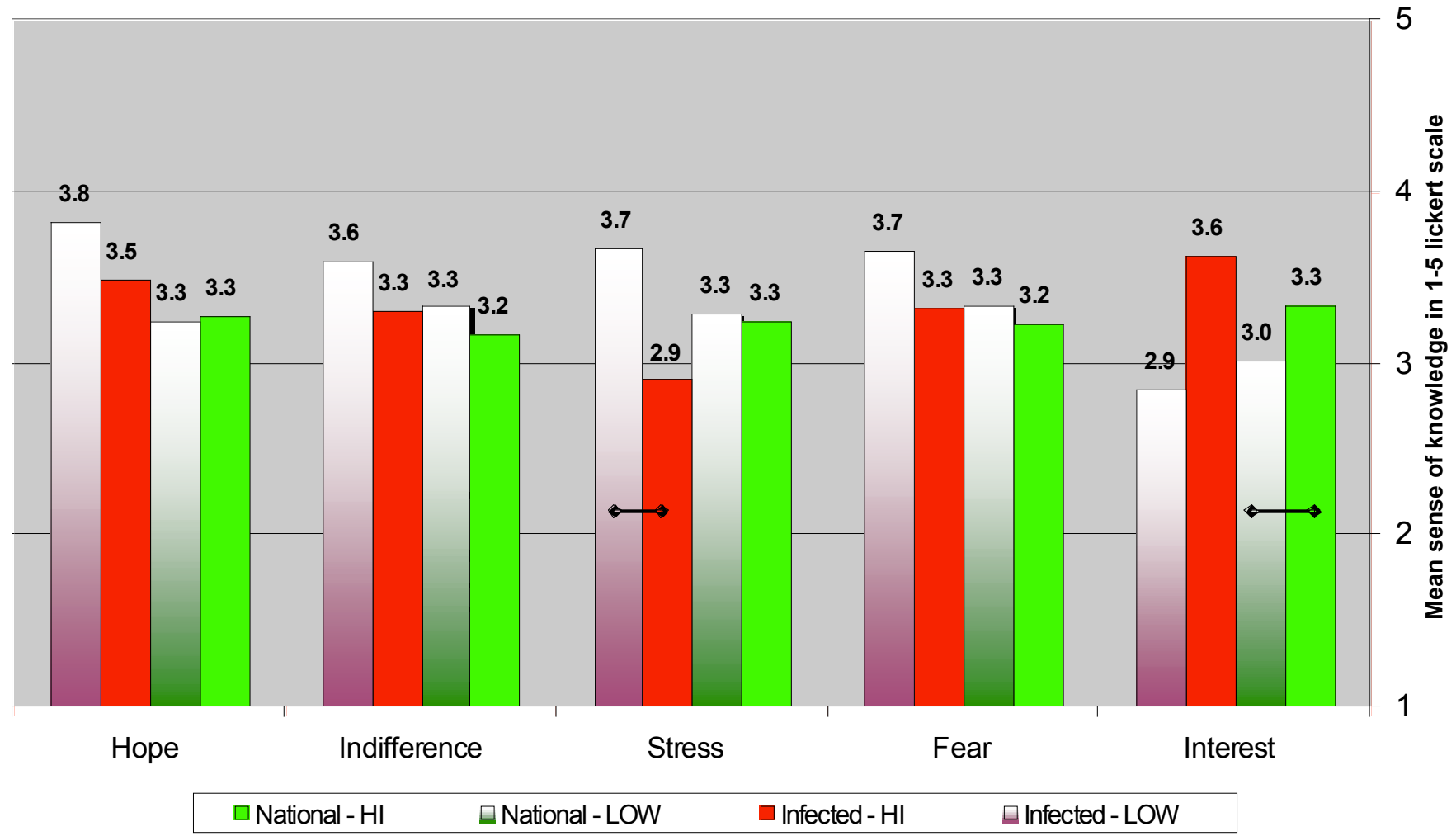

Fig. (2). The effect of feeling and interest on the sense of knowledge concerning bird flu among a representative randomly selected samples of adults in the infected areas and non infected areas in Israel. 
The psychological responses to an impending epidemic disease outbreak were studied during several outbreaks. Curson [12] drew a temporal and epidemiological framework. The process of the epidemic in the eyes of the individual and the society may begin in overseas cases and isolated local cases that may give rise to denial/disbelief reactions. Sometimes an increase in the number of local cases can produce a degree of shock and may lead to the perception of the epidemic as startling and crisis-provoking. In that phase, the escalation of emotional reactions begins. Half of the healthy residents in Hong Kong assessed their mental health as being worse because of SARS [13]. Psychological stress was prevalent throughout different phases of the epidemic. A sizable portion of the population felt horrified, apprehensive, or helpless because of the SARS epidemic in Hong Kong. In the Ovine Johne's disease [14] emotions, such as, anger, denial, hostility and anxiety, were components of the response. The SARS outbreak in Toronto caused a significant psychological impact on the city's population and in the other Canadian provinces, as well as in the United States [8]. In the case of the "mad cow" disease outbreak in Britain [15] the public reaction was highly emotional. Early efforts by authorities to play down the hazards only increased anxiety. Life after the foot and mouth disease epidemic in Britain was accompanied by distress and fear of a new disaster [16]. The majority of women in Hong Kong who discussed their emotions regarding bird flu mentioned their sense of personal fear [17]. The importance of fear was emphasized by some authors by the statement that "psychological fear is more fearful than the disease itself" [18].

The importance of knowledge in cases of epidemic diseases was mentioned in a few papers. Rumor surveillance during avian influenza outbreak is important to enhance a timely dissemination of accurate information in order to reduce misunderstanding and unwarranted concern [19]. In the SARS outbreak in Hong Kong, Lau et al. [13] pointed out that in the absence of confirmed "top-down" official information, the general public apparently formed their own attitudes in a "bottom-up" manner. Similarly, laypersons in Hong Kong, Taiwan, and Toronto used "naïve knowledge models" that were either incomplete or faulty in conceptualizing the symptoms, threat, spread, and prevention of SARS. In Toronto and United States, the public was generally knowledgeable about the ways in which the SARS can spread [8].

Avoidance behaviors are expected during an outbreak of avian flu [5]. The high prevalence of unconfirmed beliefs about modes of transmission for avian influenza may cause unwarranted panic and overreactions during an outbreak [6]. Perceptions of risk from buying live chicken were moderate in Hong Kong, but sickness anxieties did not predict buying or touching habits [7].

In a recent study, we analyzed the differences between the affected area and the nationwide population in public emotions, interest, sense of knowledge and compliance of the population with the authorities' instructions. The interest in bird flu and the sense of knowledge were higher in the affected area compared to the nationwide population. A misconception of a high human-to-human transmission rate was common in the nationwide population compared to the affected area. The levels of stress and fear perception were lower in the affected area compared to the nationwide population [10]. The level of interest, and hope correlated with a higher level of compliance in the nationwide population. The sense of knowledge correlated with a higher level of compliance, both in the affected area and in the nationwide population. The level of indifference correlated negatively with the level of compliance in the nationwide population [11].

In this study, we report significant differences in the public's sense of knowledge as a function of the source of information, emotions and interest during the early phase of a disease outbreak of avian influenza. The information that there were significant differences between users of different kinds of mass media and among people reporting different levels of emotions is important for authorities because it enables them to direct their efforts toward raising the public's sense of knowledge concerning the disease during the early phase of the outbreak, which might help to improve the compliance of the population with authorities' instructions and therefore, reduce disease spread and enable better control of the outbreak.

This study has several limitations. First, it was conducted using telephone surveys and some households may not have been included. However, in Israel almost all households have telephones. Because attitudes and perception measures may change over time, especially when circumstances change (e.g., during the early phase of a new infection) telephone surveys have the advantage over household surveys of collecting data within a short period. Second, the response rate was not very high. However, it was similar to other previously published studies [5-7]. Third, a telephone survey might exclude cell-phone only households. However, this is very rare in Israel. Forth, the cross-sectional nature of the data precludes establishing directionality. Fifth, the small sample size in the affected area prevented us from reaching significant differences between the groups, in spite of the fact that a trend of difference in the sense of knowledge was found in relation to some of the parameters in the affected area. Finally, the implications of the study were limited to the early phase of an avian influenza outbreak and further studies are needed in order to gain a more complete understanding of population behavior in different parts of a country during later phases of an outbreak.

We conclude that the sense of knowledge of the population concerning avian influenza during the early phase of the disease outbreak correlates with the source of information and with stress perception. During the early phase of a human avian influenza epidemic, there will be a need to raise the public's sense of knowledge concerning the disease in order to enhance compliance with the authorities' instructions. Psychological preparedness of the community is essential and the use of nationwide population education, especially during the early phase of an outbreak is needed. Authorities must plan how to use the different types of mass media effectively in order to increase the population's sense of knowledge concerning the disease, which might contribute to enhancing its compliance with authorities' instructions and thus improve control of a disease outbreak.

\section{ACKNOWLEDGEMENT}

The authors are indebted to Faye Schreiber, MS for the helpful revision to the manuscript. 


\section{REFERENCES}

[1] World Health Organization; WHO Activities in avian influenza and pandemic influenza preparedness, January-December 2006 [cited 2006 Nov 16]. Available from http: //www.who.int/csr/disease/avi an influenza/WHOactivities avian influenza/ en/index.html

[2] World Health Organization, Ten things you need to know about pandemic influenza. Last update Oct 14, 2005. [cited 2006 Nov 16]. Available from http: //www.who. int/csr/disease/influenza/pan demic10things/en/

[3] World Health Organization, Cumulative number of confirmed human cases of avian influenza, 2007 (10.9.08), www.who.it. [cited 2006 Nov 16]. Available from http: //www.who.int/csr/diseas e/avian influenza/country/cases table $200809 \quad$ 10/en/index.html

[4] Leung GM, Lam TH, Ho LM, et al. The impact of community psychological responses on outbreak control for Severe Acute Respiratory Syndrome in Hong Kong. J Epid Comm Health 2003; 57: 857-63.

[5] Lau JT, Kim JH, Tsui H, Griffiths S. Perceptions related to human avian influenza and their associations with anticipated psychological and behavioral responses at the onset of outbreak in the Hong Kong Chinese general population. Am J Infect Control 2007; 35(1): 38-49.

[6] Lau JT, Tsui H, Kim JH, Griffiths S. Perceptions about status and modes of H5N1 transmission and associations with immediate behavioral responses in the Hong Kong general population. Prev Med 2006; 43(5): 406-10.

[7] Fielding R, Lam WW, Ho EY, Lam TH, Hedely AJ, Leung GM. Avian influenza risk perception, Hong Kong. Emerg Infect Dis 2005; 11(5): 677-82.

[8] Blendon RJ, Benson JM, DesRoches CM, Raleigh E, Taylor-Clark $\mathrm{K}$. The public's response to severe acute respiratory syndrome in Toronto and the United States. Clin Infect Dis 2004; 38(7): 925-31.

[9] Seng SL, Lim PS, Ng MY, Wong HB, Emmanuel SC. A study on SARS awareness and health-seeking behavior - findings from a sampled population attending national healthcare group polyclinics. Ann Acad Med Singapore 2004; 33(5): 623-29.

[10] Peltz R, Avisar SG, Bar-DayanY. Differences in public emotions, interest, sense of knowledge and compliance between the affected area and the nationwide general population during the first phase of a bird flu outbreak in Israel. J Infect 2007; 55: 545-50.

[11] Bar-Dayan Y, Avisar SG, Peltz R. Compliance with the authorities instructions during the early phase of a bird flu outbreak in Israel. 2007; submitted.

[12] Curson P. Paradise delayed: Epidemics of infectious disease in the industrialized world. In: Clarke J, Curson P, Kayastha S, Eds. Population and Disaster, Institute of British Geographers. Cambridge: Blackwell; 1989. pp. 159-79.

[13] Lau JT, Yang X, Pang E, Tsui HY, Wong E, Wing YK. SARSrelated perceptions in Hong Kong. Emerg Infect Dis 2005; 11(3): 417-24.

[14] Hood B, Seedsman T. Psychological investigation of individual and community responses to the experience of ovine John's disease in rural Victoria. Aust J Rural Health 2004; 12(2): 54-60.

[15] Granot H. Facing catastrophe: mad cows and emergency policymaking. Int J Mass Emerg Disasters 1999; 17(2): 161-84.

[16] Mort M, Convery Y, Baxter J, Bailey C. Psychosocial effects of the 2001 UK foot and mouth disease epidemic in a rural population: qualitative diary based study. BMJ 2005; 331(7527): 1234. Epub 2005 Oct 7.

[17] Joffe H, Louise L. Social representation of food risk: the Hong Kong avian bird flu epidemic. J Health Psychol 2004; 9(4): 517-33.

[18] Cheng C, Cheung M. Psychological responses to outbreak of Severe Acute Respiratory Syndrome: a prospective, multiple time point study. J Pers 2005; 73: 261-85.

[19] Samaan G, Patel M, Olowokure B, Roces M, Oshitani H, WHO outbreak response team; Rumor surveillance and avian influenza H5N1. Emerg Infect Dis 2005; 11(3): 463-6. 